\title{
THE DISTRIBUTION OF SEPARATIONS OF WIDE BINARIES OF DIFFERENT AGES
}

\author{
Arcadio Poveda ${ }^{1}$ and Christine Allen ${ }^{1}$ \\ RESUMEN
}

Con el propósito de entender cómo evoluciona la distribución de separaciones de los sistemas dobles y múltiples hemos estudiado varios grupos de muy distintas edades. Reseñamos y complementamos nuestro trabajo previo para los siguientes grupos de binarias: (a) Las binarias abiertas del IDS. (b) Los pares de movimiento propio común de Luyten (LDS). (c) Nuestro catálogo de binarias del entorno solar, dividido en dos grupos: las más jóvenes y las más viejas. (d) Los pares de movimiento propio común del cúmulo de la Nebulosa de Orión. (e) Nuestra muestra de binarias de alta velocidad y baja metalicidad. Encontramos que desde los grupos más jóvenes (cúmulo de la Nebulosa de Orión) hasta los más viejos (disco grueso y halo galácticos), la distribución de semiejes mayores es la de Oepik. Esta es válida desde $a$ igual a $60 \mathrm{AU}$ hasta $a_{m}(t)$, donde $a_{m}(t)$ es un semieje máximo que depende de la edad de la binaria y de la densidad y velocidad de los perturbadores masivos que ésta encuentra en su recorrido por la Galaxia. Los encuentros gravitacionales intensos en un proto-cúmulo producen binarias con la distribución de Oepik; ésta se ve truncada a separaciones pequeñas por los discos circunestelares y las binarias cerradas, y a separaciones grandes por los encuentros con objetos masivos.

\section{ABSTRACT}

For the purpose of understanding how the distribution of major semiaxes of double and multiple stars has evolved from the earliest ages up to the ages of the oldest systems, we have studied a number of different groups of such systems. We review and expand our previous work on the following groups of stars: (a) The wide binaries in the IDS; (b) Luyten's common proper motion pairs (the LDS catalogue). (c) Our catalogue of nearby wide binaries divided into two groups: the youngest and the oldest; (d) The common proper motion pairs in the Orion Nebula cluster; (e) Our sample of high velocity, low metallicity binaries that represents a population of very old systems. From the very young binaries (Orion Nebula Cluster) to the oldest (thick disk and halo), all groups show a distribution of major semiaxes following Oepik, i.e., $f(a) d a \propto d a / a$ (where $a$ is the major semiaxis), valid over an interval of $a$ between $60 \mathrm{AU}$ and $a_{m}(t), a_{m}(t)$ being a maximum semiaxis which depends on the age of the binaries as well as on the number density and velocities of the massive objects they encounter as they travel in the Galaxy. Strong gravitational encounters in recently formed multiple systems were found to produce an Oepik distribution, which at small separations ( $a \leq 40-60 \mathrm{AU})$ gets truncated by circumstellar disks and close binaries, and at large separations gets depleted by encounters with massive objects.

\section{Key Words: BINARIES: GENERAL - BINARIES: STATISTICS - STELLAR DYNAMICS}

\section{INTRODUCTION}

The observed present-day distribution of separations of wide double and multiple stars contains important information about the conditions of star formation, and about the subsequent dynamical evolution of the orbital parameters. Of particular interest is the distribution of separations (major semiaxes), which appears to reveal two different mechanisms of star formation. Wide binaries $(a>40-60 \mathrm{AU})$ are particularly affected by gravitational perturbations produced by the remaining members of the multiple system in which they were born. Later on, as they

\footnotetext{
${ }^{1}$ Instituto de Astronomía, Universidad Nacional Autónoma de México.
}

travel through the Galaxy, they encounter massive objects (molecular clouds, spiral arms, black holes, etc.), which further disturb and deplete the original distribution of major semiaxes.

Our interest in the distribution $f(a)$ of major semiaxes in wide binaries goes back to the late sixties. First, we performed numerical simulations to explain the formation of runaway stars by strong interactions in a few-body cluster; the large kinetic energy of the runaway star(s) is compensated by the binding energy of the binary(ies) formed during the close interactions (Poveda et al. 1967). Second, we determined the dissociation time of a class of binaries, with the aim of clarifying the inconsistencies in 
the dissolution times given by Oort, Chandrasekhar and Ambartsumian (Cruz-González \& Poveda, 1971, and references therein). A comparison of the distribution $f(a)$ of the binaries formed in the strong few-body interactions with the corresponding distribution for field stars gives support to our model of runaway star formation and provides insight into the mechanism of double star formation. (Allen \& Poveda 1968; Allen 1968)

\section{CLOSE AND WIDE BINARIES}

The frequency distribution of the masses of the components, the multiplicity of field stars, the relation of close binaries to wide binaries, the distribution of major semiaxes for each type, and the kinematics of various groups of binaries, are all characteristics that keep memory of the process of star formation and of the subsequent dynamical interactions, both internal and external.

In the present paper we are concerned with the distribution of major semiaxes of wide binaries; In fact, several functions have been proposed in the past to describe the distribution of the periods, major semiaxes, or separations. We identify two main distributions: Oepik's (1924) frequency distribution of separations $f(s) \propto 1 / s$, and Kuiper's (1942) distribution, which is expressed as a Gaussian in $\log a$ (or $\log P$ ). More recently, Duquennoy and Mayor (1991), based on a large sample of high precision radial velocities for stars in Gliese et al.'s catalogue (1991), found the frequency distribution of $\log P$ to be a Gaussian with a maximum at $\log P$ (days) $\approx 4.5$. In contrast, Close et al. (1990) find, for a sample of nearby binaries that $f(s)=k s^{-1.3}$, which resembles Oepik's relation within the uncentainties of the data.

Note that there are several equivalent ways to express Oepik's relation:

$$
\begin{aligned}
f(s) \propto s^{-1}, & f(a) \propto a^{-1} \\
N(s) \propto \log s, & N(a) \propto \log a .
\end{aligned}
$$

Here, $f$ represents the frequency distribution and $N$ the cumulative distribution.

In a classical paper, Abt and Levy (1976) showed that the mass frequency distribution of the secondaries of a sample of binaries with $G$-type primaries followed van Rhijn's luminosity function (Salpeter's mass function) for those binaries with periods larger than about 100 years ( $a \geq 25 \mathrm{AU}$ ), while for those binaries with smaller periods the distribution of masses of the secondaries was more like $f(m) \propto m^{1 / 2}$. That is, while for wide binaries the less massive the secondary the more frequent it is, for the short period ones the smaller the mass of the secondary the less frequent it is. This important result has been later confirmed by other authors (see for instance Mazeh and Goldberg 1992, and Mazeh et al. 1992).

The fact that the secondaries have two different mass distributions provides a strong indication that we are facing two different processes of star formation, one for the short-period binaries and another for the wide binaries. Since wide binaries follow Salpeter's mass distribution, we take this as evidence that these binaries are the result of the random pairing of two independent condensations (stars) each one extracted from the Salpeter mass distribution. On the other hand, the close binaries, because of the small scale of the major semiaxes, are entirely compatible with the scale of preplanetary disks, and thus they are likely to be the result of fragmentation of circumstellar disks. Numerical simulations of disk fragmentation (Boss 1995, Bate 2004, and references therein) support this explanation for the formation of close binaries. Having a different mass distribution and hence a different origin, each one of these groups of binaries is likely to follow a different distribution of separations. For example, binaries that stem from disk fragmentation cannot have major semiaxes larger than the radius of preplanetary disks (at most a few hundred AU), while binaries that result from independent condensations in a dense cloud may have much larger major semiaxes (of the order of Jeans' scale in the cores of molecular clouds, i.e., tens of thousands of $\mathrm{AU}$ ); on the other hand, binaries stemming from independent condensations may not be able to form (or survive) at separations smaller than a few tens of $\mathrm{AU}$, because they may be destroyed by the process of disk formation and fragmentation into stars.

Because of our interest in the dynamical evolution of $f(a)$ induced by gravitational interactions with massive field objects we have centered our attention on Abt and Levy's wide binaries (i.e. $a \geq 25$ $\mathrm{AU})$

In order to pursue the above task we embarked in a long range investigation on the distribution $f(a)$ for various groups of stars, some of them of different ages.

To establish the distribution of major semiaxes for wide binaries requires data for a large number of binaries for which orbital elements are known. Unfortunately, the periods of wide binaries (visual and common-proper-motion pairs) are so long that orbit determination is possible only for a few of them, and therefore no major semiaxes are available for the great majority.

In order to circumvert the above limitations, we 


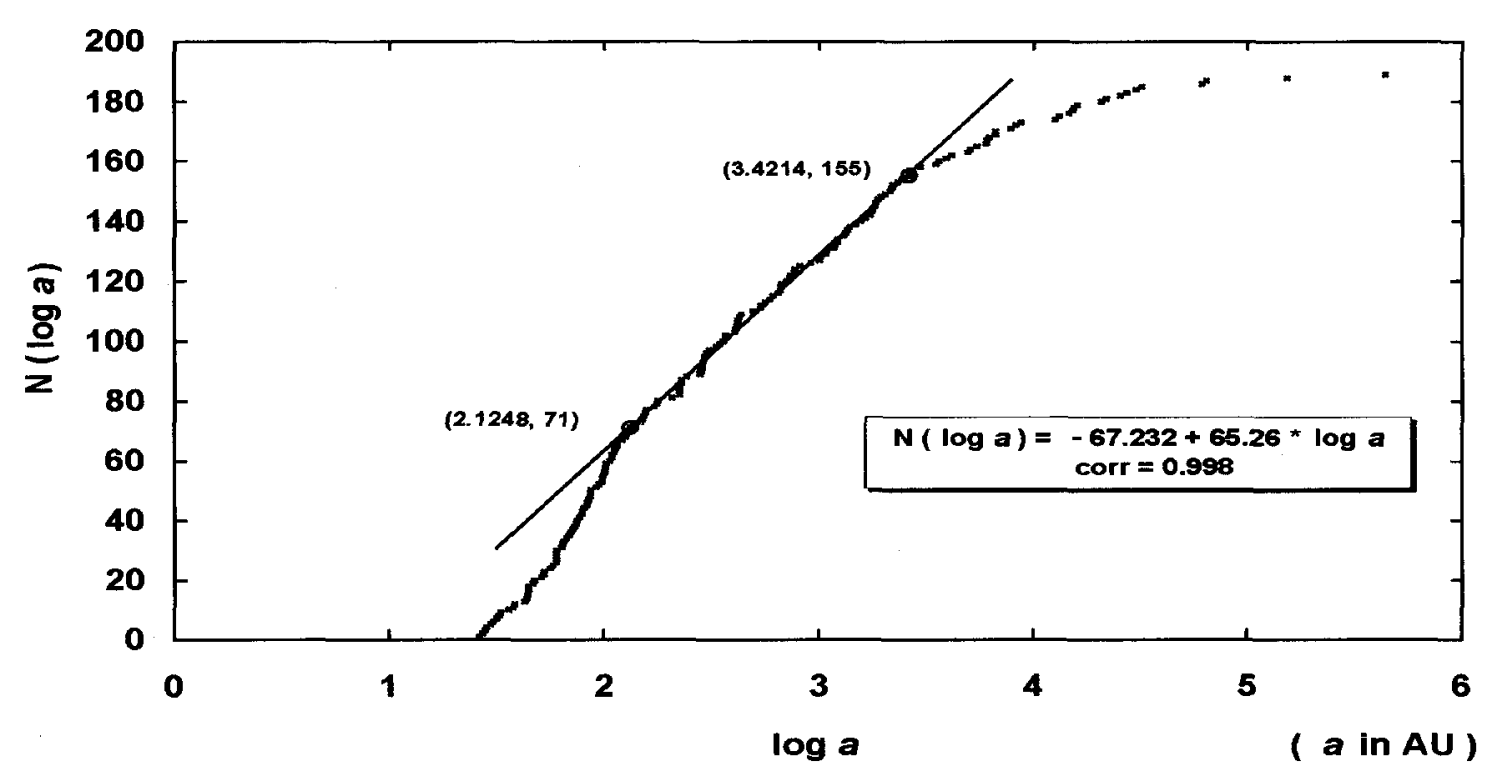

Fig. 1. Cumulative distribution of $\log a$ for the PHACL Catalogue of Nearby Wide Systems. $M_{V} \leq 9$, luminosity class IV or V. The number of binaries plotted is $N=189$. See text for more details.

will take advant tge of the following theorem, valid in a statistical sense. For wide binaries of known distances, Couteau (1960) derived the following relation:

$$
E(\log a)-E(\log s)=0.146
$$

where $a$ is the major semiaxis, $s$ the projected separation on the sky of the components of a binary, and $E$ the expectation value of the variable in parentheses. This relation was derived on theoretical grounds. Couteau tested its observational validity against a catalogue of 410 orbits and found the constant to be 0.150 . It is not too different from the empirical relation derived earlier by Kuiper (1942), where the constant had a value of 0.11 .

Couteau's relation allows us to obtain a statistical estimation of the major semiaxes of a binary from the observed angular separation $s$ of the components. When the distance to a given pair is known, the angular separation can be converted to a linear separation (in $\mathrm{AU}$ ), and hence the estimate of $a$ is also known in AU. We have determined the expected major semiaxes in $\mathrm{AU}$ for a large number of wide binaries both in the solar vicinity, and in galactic clusters.

In the following we shall use either the projected separations $s$, or the expected major semiaxes $\langle a\rangle$ interchangeably, since they are equivalent for the present discussion. ${ }^{2}$

\footnotetext{
${ }^{2}$ We will take $a$ to mean $a=\langle a\rangle=$ antilog $\left[\log \left(s^{\prime \prime} / \pi\right)+0.146\right]$
}

There are, however, quite a number of binaries for which no distances are known, for instance, binaries from the IDS, from the WDS, or from the LDS. For these objects we can still investigate the form of the frequency distribution of separations by taking advantage of a theorem first suggested by Ambartsumian (1937).

If the distribution $f(s)$ of the projected angular separations of a group of binaries located at very different distances from the observer is $f(s) \propto s^{-1}$, then the distribution of their "true" projected separations $r$ has also the form $f(r) \propto r^{-1}$. It can be shown that this theorem is valid more generally, for other values of the exponent (Poveda 2004).

With the help of the above theorems we have been able to determine the form of the distribution of separations of binaries from catalogues like the IDS and the LDS (Poveda 1988).

The groups of binaries that we have investigated are the following:

1. Binaries in the IDS and the LDS.

2. Wide binaries and multiples in the solar vicinity, subdivided into (a), the youngest and (b), the oldest systems.

3 . Wide binaries in young clusters.

4. High velocity metal poor wide binaries.

\section{BINARIES IN THE IDS AND THE LDS}

A study of the 51,000 binaries in the IDS catalogue, after elimination of a large number of probable optical pairs (Poveda et al. 1982), has shown 


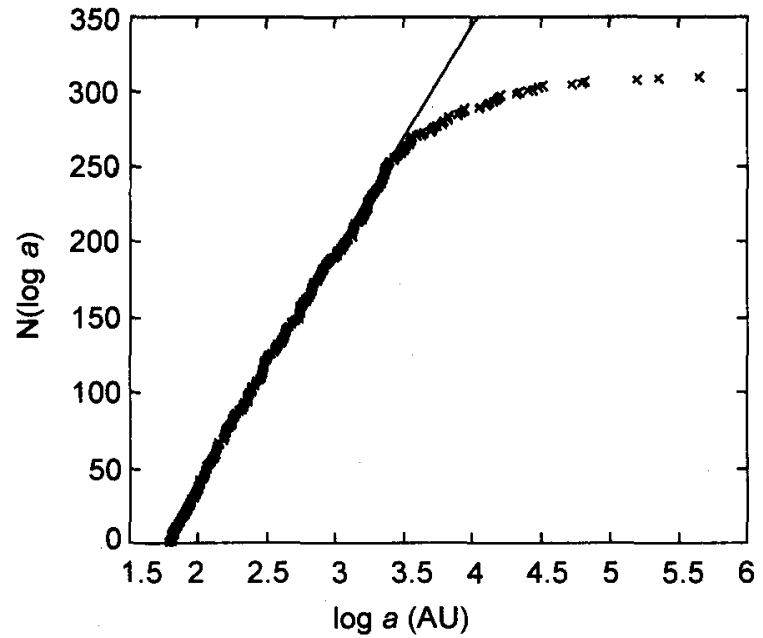

Fig. 2. Comparison between Oepik's cumulative distribution and the cumulative numbers of $\log a$ for all the binaries in the PHACL Catalogue, in the interval $60 \mathrm{AU}$ $<a<2965 \mathrm{AU}$. The $\mathrm{K}-\mathrm{S}$ test gives a $\mathrm{Q}$ value of $\mathrm{Q}=$ 0.95793 (260 binaries)

that the distribution of separations has the form $f(s) \propto s^{-1}$ (Poveda 1988). The close companions of the triples in the same sample also follow Oepik's distribution, while the distant companions of the same triples show a much slower decay with separation.

The Luyten (1969) Double Star Catalogue is an alternative source of binaries which differ from those that prevail in the IDS because most are intrinsecally faint and, more important, because the physical reality of the pairs is assured by their common proper motions. For the purpose of studying the distribution of separations, we have selected the 562 pairs found by Luyten in the Palomar plates in the cap north of $+60^{\circ}$ declination, which Luyten recommends for statistical studies.

For this sample of the LDS catalogue, comprising a completely different set of binaries, we found that Oepik's law is again satisfied (Poveda 1988).

\section{WIDE BINARIES IN THE SOLAR VICINITY}

A search for wide binaries in Gliese's catalogue (Poveda et al. 1994, henceforth PHACL) produced a list of 334 wide binaries and multiples for which expected major semiaxes in AU are available; moreover, for these systems, an age classification has been given, thus making it possible to compare the distribution of $a$ for the youngest and for the oldest systems. From the analysis of the solar vicinity binaries we found not only that the distribution of expected major semiaxes follows Oepik's function, but also that the maximum $a_{m}(t)$ for which Oepik's distribution holds is $a_{m}(t)=2400 \mathrm{AU}$ for the oldest systems, and $a_{m}(t)=8000 \mathrm{AU}$ for the youngest.

In fact, since Gliese \& Jahreiss' Catalogue is volume-complete to $M_{V} \cong 9$ (Gliese, Jahreiss \& Upgren 1986) and our search for companions to Gliese's stars is also complete to the limiting magnitude of the NLTT, we have first made an analysis of the frequency distribution of separations for all the binaries (and multiples) in the PHACL catalogue with primaries $M_{V} \leq 9$.

In Figure 1 we show the distribution $\mathrm{N}(\log a)$ vs. $\log a$. Each cross corresponds to a binary; for each set of increasing values $\log a_{1}, \ldots, \log a_{i}$ we have counted $\mathrm{N}\left(\log a_{i}\right)=1,2, \ldots \mathrm{N}$ and fitted by leastsquares the best straight line. By trial and error we have found the best straight line that fits the set of data $\left[\mathrm{N}\left(\log a_{i}, \log a_{i}\right]\right.$ for the largest number of binaries in the widest interval in $\log a$. The "best straight line" and the "best interval" $a_{i}, a_{n}$ were determined by the Kolmogorov-Smirnov test.

In Figure 1 we show, among the many least square fits that can be tried, the best straight line in the interval $2.12<\log a<3.42 ;(71 \leq \mathrm{N} \leq 155)$. The equation of this fit and its correlation coefficient are also shown in Figure 1. From this fit, based on a volume-complete sample of wide binaries, we can see that Oepik's distribution is a very good representation of the distribution $f(a)$ in the interval of major semiaxes $133 \mathrm{AU}<a<2640 \mathrm{AU}$.

Having established that a volume-complete sample of wide binaries can be represented by the distribution $f(a) \sim a^{-1}$ we felt confident to extend the same type of analysis to the whole PHACL catalogue. Figure 2 shows the best regression in the interval $60 \mathrm{AU}<a<2965$. The Kolmogorov-Smirnov test (K-S) gives the probability $Q=0.96$ that the set of 260 binaries in the above mentioned interval of major semiaxes have, in fact, been extracted from a frequency distribution $f(a) \sim 1 / a$. The binaries (and multiples) in our catalogue were then grouped into young and old according to whether at least one of the components shows indications of youth, like spectral type earlier than F5 V, chromospheric activity, $V \sin i$ larger that $6 \mathrm{~km} \mathrm{~s}^{-1}$, membership to young moving clusters, etc. (Poveda et al 1994). To maximize the age contrast, a selection of the youngest and the oldest binaries was examined to see if the largest major semiaxis $a_{m}$ which fits Oepik's distribution, depends on the age of the binaries.

Figure 3 shows, as before, the best fit to the distribution $N(\log a)$, for the oldest binaries; in the in- 




Fig. 3. Comparison between Oepik's cumulative distribution and the cumulative numbers of $\log a$ for the oldest binaries in the PHACL Catalogue, in the interval $60 \mathrm{AU}$ $<a<2409 \mathrm{AU}$. The $\mathrm{K}-\mathrm{S}$ test gives a value of $\mathrm{Q}=0.99 \ldots$ (63 binaries)

terval $60 \mathrm{AU}<a<2409 \mathrm{AU} .63$ binaries give an excellent agreement with Oepik's distribution; the K-S test gives the probability $Q=0.99 \ldots$

The distribution $N(\log a)$ for the sample of the youngest binaries is shown in Figure 4 . Here we can confirm again that this sample is very well represented by Oepik's distribution in the interval $69 \mathrm{AU}$ $<a<7862 \mathrm{AU}$.

The maximum major semiaxis for which Oepik's distribution holds is much larger for the youngest binaries $\left(a_{m}=7862 \mathrm{AU}\right)$ than for the oldest $\left(a_{m}=\right.$ $2409 \mathrm{AU})$

An analysis of the velocity dispersion for these two age groups, using Wielen's (1977) relation yields average ages of 7 and 2.5 Gigayears for the oldest and the youngest binaries, respectively.

Weinberg et al. (1987) have modeled the evolution of binaries subject to the perturbations by encounters with molecular clouds. In Figure 5, adapted from their paper, it can be seen that systems with separations smaller that $2000 \mathrm{AU}$ are practically unperturbed after $10^{10}$ years, while systems with original major semiaxes of 13,000 AU have a probability of survival of only 0.1 .

From the above, we can conclude that within the mixture of stars in the solar vicinity the primeval distribution of major semiaxes follows Oepik's, and has remained intact up to $2400 \mathrm{AU}$ throughout the age of the galactic disk; on the other hand, binaries

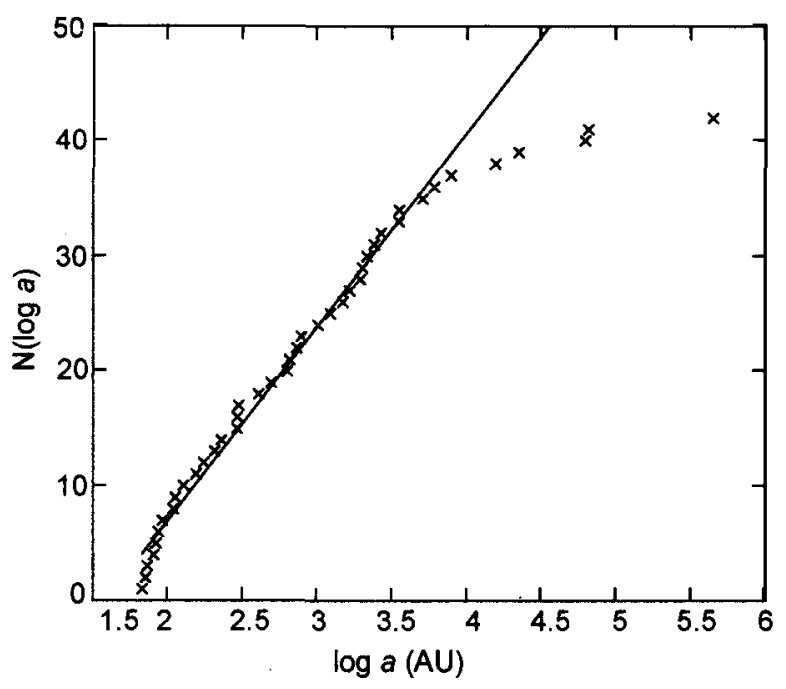

Fig. 4. Comparison between Oepik's cumulative distribution and the cumulative numbers of $\log a$ for the youngest binaries in the PHACL catalogue in the interval $60 \mathrm{AU}$ $<a<7862 \mathrm{AU}$. The $\mathrm{K}-\mathrm{S}$ test gives a $\mathrm{Q}$ value of $\mathrm{Q}=$ 0.95 ( 37 binaries)

with original major semiaxes of the order of 13,000 $\mathrm{AU}$ have practically disappeared after 10 gigayears. This explains the leveling off of the cumulative distribution N( $\log a)$ for $a>2400 \mathrm{AU}$ for the oldest binaries; for the youngest binaries in the solar vicinity, Oepik's distribution holds up to much larger separations, as can be seen in Figure 4.

It is interesting to note in this context that binaries extracted from the Hipparcos Catalogue (Prieto, Ling \& Pilar, 2004), which mostly belong to the extended solar vicinity, also follow Oepik's distribution.

\section{WIDE BINARIES IN YOUNG CLUSTERS. THE ORION NEBULA CLUSTER.}

For the purpose of identifying a group of wide binaries in a very young cluster $\left(T \leq 10^{6}\right.$ years $)$ and also to get a closer look at the "primeval" distribution of separations, we have conducted a search for common proper motion companions among the nearest neighbors of each one of the Orion Nebula Cluster stars, for which Jones \& Walker (1988) have determined proper motions (Poveda \& HernándezAlcántara, 2003). Each one of these pairs was put through a selection procedure in order to obtain a sample with a large expected number of physical systems. We have identified 68 such pairs with projected separations between 2000 and 33,000 AU. As shown in Figure 6, the distribution of expected major semiaxes $f(a)$ follows Oepik, i.e. $f(a) d a \propto d a / a$ up to $30,000 \mathrm{AU}$. 


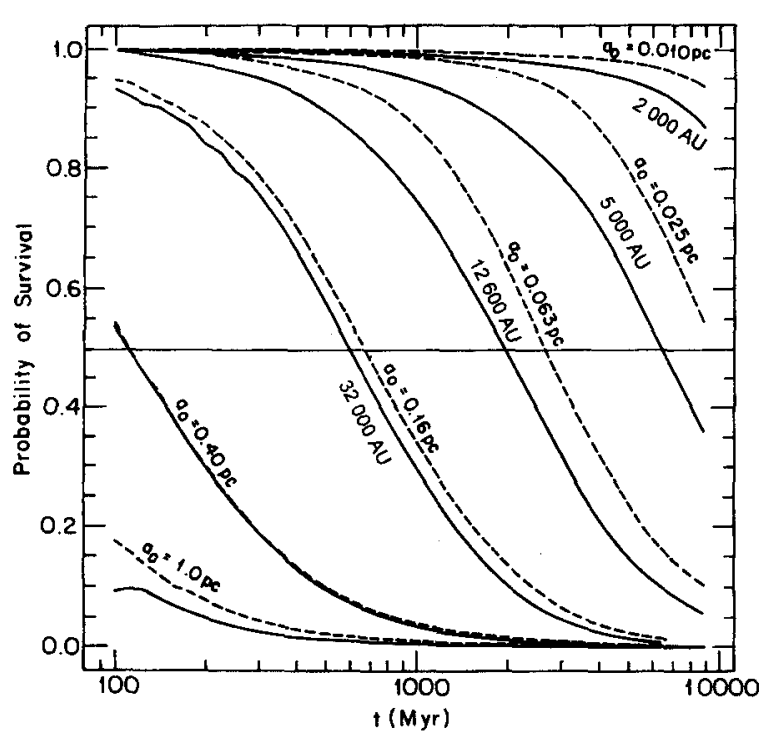

Fig. 5. Probability of survival as a function of age for binaries of different major semiaxes (Weinberg et al. 1987). For further details, see references.

\section{HIGH-VELOCITY, METAL-POOR BINARIES.}

Allen, Poveda \& Herrera (2000) have searched for common-proper-motion companions to the more than 1200 high-velocity, metal-poor stars observed by Schuster and Nissen $(1988,1989)$. The comparison of the proper motions of the above high-velocity stars with Luyten's NLTT stars has yielded 122 wide binaries belonging mostly to the galactic thick disk or halo. The distribution of major semiaxes for this group of binaries again follows Oepik's relation, but now up to $a_{m}(t) \geq 10,000 \mathrm{AU}$. The binaries were divided into two groups according to the characteristics of their galactic orbits. The most disk-like stars spend 100 percent of their lifetimes within the galactic disk $(|z|<500 \mathrm{pc})$. The most halo-like stars spend only 26 percent of their lifetimes within the galactic disk. In Figures 7 and 8, we display the comparison of the cumulative distribution of the logarithms of the major semiaxes of both groups of high-velocity, metal-poor stars against Oepik's distribution. Note that the data begin to depart from Oepik's relation at $a \geq 8000 \mathrm{AU}$ for the disk-like binaries, whereas Oepik's relation holds up to $a \leq$ 20,000 AU for the most halo-like binaries.

The survival of Oepik's distribution past 10,000 $\mathrm{AU}$ for this population of very old binaries is to be understood as due to the fact that these binaries spend most of their life outside the galactic disk, where massive perturbers are mostly located; moreover, when they encounter them in the disk, they

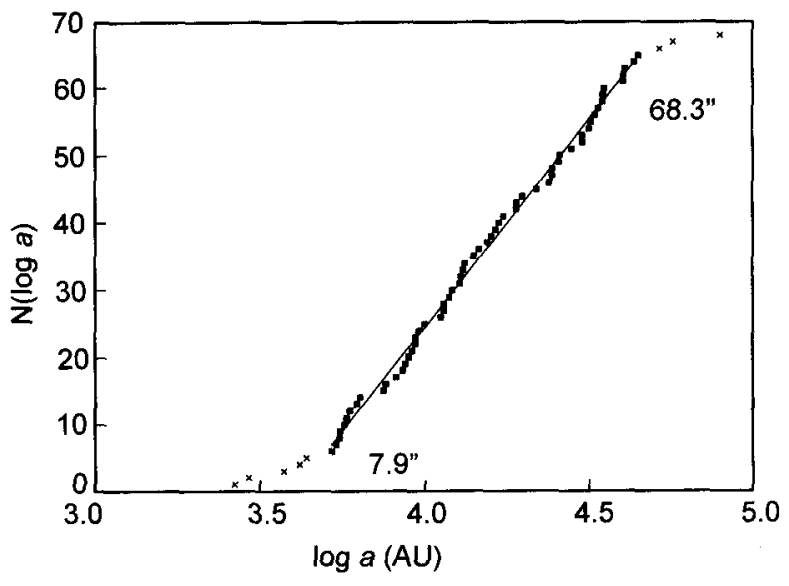

Fig. 6. Cumulative distribution $N(\log a)$ of the projected major semiaxes of the common proper motion binaries in the Orion Nebula Cluster vs. $\log a$

do so at large velocities, which do not favor energy exchánges.

\section{DISCUSSION AND CONCLUSIONS}

In the present paper we have examined a variety of wide binaries in different groups and environments, with ages ranging from extremely young $\left(10^{6} \mathrm{y}\right)$ to very old $\left(14 \times 10^{9} \mathrm{y}\right)$. We have consistently found that they follow Oepik's relation. Moreover, the maximum major semiaxes $a_{m}(t)$ change with age in agreement with the expectations, in the sense that the older the binaries, the smaller $a_{m}(t)$. Even the high-velocity, metal-poor binaries confirm this behavior, because the larger value found for the maximum major semiaxes, $a_{m}(t) \geq 10,000 \mathrm{AU}$, can be understood as a result of the fact that most of these objects have orbits that take them away from the galactic disk, where the number density of perturbers (molecular clouds) is largest.

When one compares the distribution $f(a)$ for the various age groups one is compelled to think that the process of star formation is such that a number of independent condensations (with a Salpeter mass frequency distribution) are formed in very dense and cold clouds in contraction; pre-stellar condensations in such a cloud will tend to fall to the center of mass. In so doing they will experiment mutual strong gravitational interactions; the net result of these interactions is to eject one or more stars with positive energy, and to form one or more binaries.

Numerical simulations performed by us many years ago (Poveda, Ruiz \& Allen 1967; Allen \& Poveda 1968; Allen 1968) were succesful in producing runaway stars as well as binaries, starting from a collapsing proto-cluster with massive condensations. We 


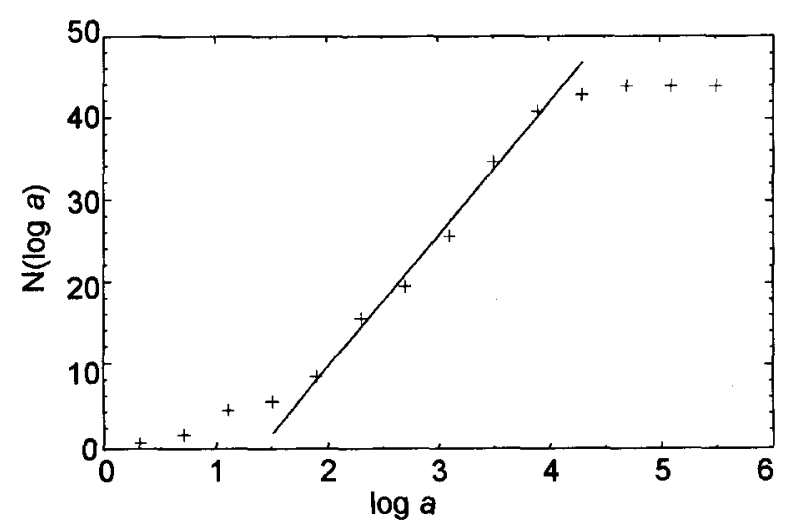

Fig. 7. Cumulative distribution of the expected values of the logarithms of the major semiaxes for Group I, the most disk-like binaries among the high-velocity metalpoor stars.

computed the orbital parameters of the binaries produced in 146 simulations of runaway and multiple star formation. Figure 9 shows the frequency distribution of major semiaxes found in those computations.

We performed these computations much before we had overwhelming evidence in favor of Oepik's distribution. In fact, in Figure 9 (adapted from Allen 1968) we may even imagine that we have the superposition of two Gaussian distributions. However, a closer look shows that the histogram is also consistent with Oepik's distribution. Actually, the horizontal line (which is the average of $f(\log a)$ in the interval $1<\log a<3$ ) falls within the error bars $\pm \sqrt{f(\log a)}$. When we display the results of these simulations as a cumulative distribution (Figure 10), we obtain a very good fit to Oepik's distribution. Moreover, the parameters we chose 35 years ago, much before our study on wide binaries in the solar vicinity, give a good representation in the interval of major semiaxes between 10 and $1000 \mathrm{AU}$. Clearly, by appropriately tuning the initial conditions, we may reproduce a distribution like that of the youngest binaries in the solar vicinity, or those in the Orion Nebula Cluster.

It is interesting to note that Valtonen (1997) found that the application of the statistical theory of three-body breakup in a cluster enviroment leads naturally to Oepik's law for the binary separations. This law was found to be valid also for finite-size bodies.

Thus we conclude that the initial conditions of star formation in very compact nebular condensations, combined with their mutual gravitational interactions, produce wide binaries with Oepik's dis-

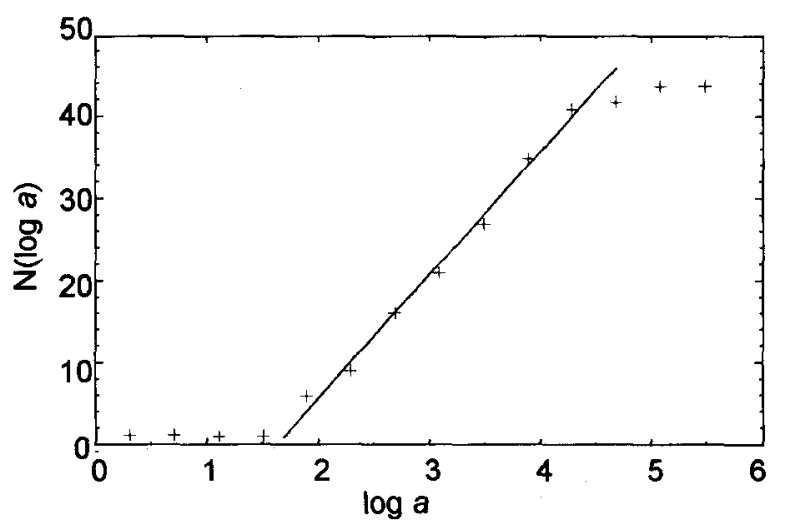

Fig. 8. Cumulative distribution of the expected values of the logarithms of major semiaxes for Group II, the most halo-like binaries among the high-velocity metalpoor stars.



Fig. 9. Frequency distribution of $\log a$ for the binaries formed in collapsing proto-stellar clusters.

tribution of major semiaxes. This distribution gets truncated at small separations by the presence of circumstellar disks; at large separations it gets depleted by the gravitational perturbations of massive objects. Figure 1 nicely illustrates this scenario.

Duquennoy \& Mayor have proposed that the distribution of the logarithms of the periods of solar vicinity binaries, both close and wide, is Gaussian. Note however that in their plane $N-\log P$, Oepik's distribution is a horizontal straight line; if we plot Oepik's distribution in Duquennoy \& Mayor's Figure 7 for major semiaxes in the interval $42 \leq a(\mathrm{AU})$ $\leq 4213$ we see (Fig. 11) that Oepik's distribution is entirely consistent with Duquennoy \& Mayor's histogram (within the error bars) for the few wide binaries (28) they plot in this interval.

From the above discussion it follows that the Gaussian representation may be the result of combining two different distributions of major semiaxes, 




Fig. 10. $N(\log a)$ vs. $\log a$ for the binaries formed in collapsing pre-stellar condensations.

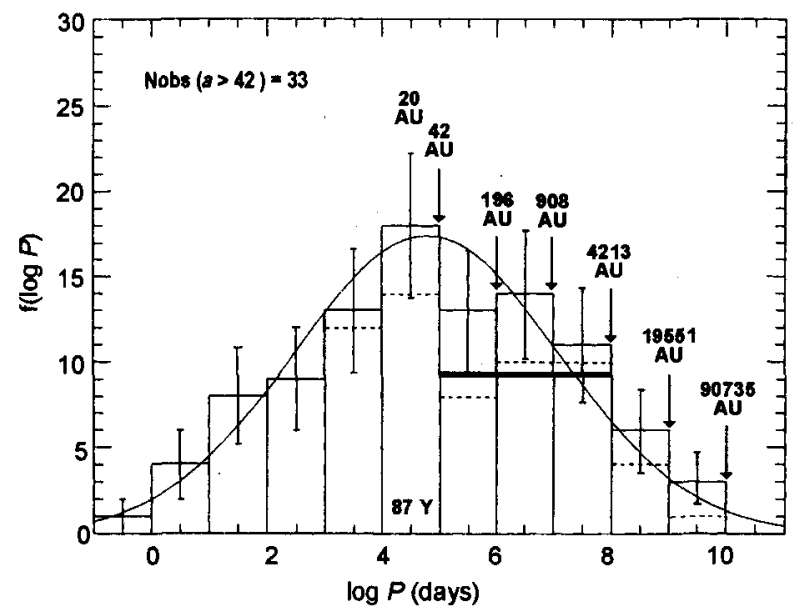

Fig. 11. Adapted from Figure 7 of Duquennoy \& Major's paper. In this plane Oepik's distribution is mapped as a horizontal segment $N=$ constant $=28 / 3$ in the interval $42 \mathrm{AU}<a<4213 \mathrm{AU}$. This segment corresponds to the cumulative distribution $\mathrm{N}(\log a)$ in the interval $60 \mathrm{AU}$ $<a<3000 \mathrm{AU}$, as displayed in Figure 2. See text for the physical interpretation of this interval.

an increasing one for close binaries and a decreasing one for wide binaries. The overlap of both distributions at about $a=25-40$ AU simulates a Gaussian distribution.

We are grateful to A. Hernández-Alcántara, for his technical support in the preparation of this paper, and to CONACYT for financial support through grant Nr. 22502E.

\section{REFERENCES}

Abt, H.A., \& Levy, S., 1976, ApJS, 30, 273.

Allen, C., 1968. "Sobre el origen de las estrellas dobles masivas", Thesis, UNAM.

Allen, C., \& Poveda, A., 1968, AJ, 73, S86.

Allen, C., Poveda, A., \& Herrera, M. A., 2000, A\&A, 356, 529.

Ambartsumian, V.A., 1937, Russ. Astron. J., 14, 207.

Bate M.R., 2004, this volume.

Boss, A.P., 1995, RevMexAA (SC), 1, 165.

Close, L.M., Richer, H.B., \& Crabtree, D.R., 1990, AJ, 100, 1968.

Couteau, P., 1960, J. des Observateurs, 43, 41.

Cruz-González, C., \& Poveda, A, 1971, Ap\&SS, 13, 335.

Duquennoy, A., \& Mayor, M., 1991, A\&A, 248, 495.

Gliese, W., Jahreiss, H., \& Upgren, A., 1986, in "The Galaxy and the Solar System", eds. Smoluchowski, R., Bahcall, J., \& Matthews, M. (Tucson: University of Arizona Press), p. 13.

Gliese, W., \& Jahreiss, H., 1991, in "Selected Astronomical Catalogs", eds. Brotzman, L.E., \& Gessner, S.E., VOL. I, NSSC, NASA, GSFC (GJ91).

Jones, B.F., \& Walker, M.F., 1988. AJ, 95, 1755.

Kuiper, G.P., 1942, ApJ, 95, 201.

Luyten, W.J., 1969, Proper Motion Survey with the 48Inch Schmidt Telescope, XXI Double Stars with Common Proper Motion, University of Minnesota, Minneapolis.

Mazeh, T., \& Goldberg, D., 1992, ApJ, 394, 592.

Mazeh, T., Goldberg, D., Duquennoy, A., \& Mayor, M., 1992, ApJ, 401, 265.

Oepik, E., 1924, Tartu Obs. Publ, 25, No. 6.

Poveda, A., Ruiz, J., \& Allen, C., 1967, Bol. Obs. Ton. Tac., 4, 86.

Poveda, A., Allen, C., \& Parrao, L., 1982, ApJ, 257, 589.

Poveda, A., 1988, Ap\&SS, 142, 67.

Poveda, A., Herrera, M. A., Allen, C., Cordero, G., \& Lavalley, C., 1994, RevMexAA, 28, 43.

Poveda, A., \& Hernández-Alcántara, A., 2003, in "Proc. of the Sixth Pacific Rim Conference on Stellar Astrophysics", eds. Cheng, K.S., Leung, K.C., \& Li, T.P. (Dordercht: Kluwer), in press.

Poveda, A., 2004, in preparation.

Prieto, C., Ling J.F., \& Pilar, M., 2004, this volume.

Schuster, W., \& Nissen, P., 1988, A\&AS, 73, 225.

Schuster, W., \& Nissen, P., 1989, A\&A, 222, 69.

Valtonen, M. 1996, in "Visual Double Stars: Formation, Dynamics and Evolutionary Tracks", eds. Docobo, J.A., Elipe, A., \& McAlister, H. (Dordercht: Kluwer), p. 241.

Weinberg, M.D., Shapiro, S.L., \& Wasserman, I., 1987, ApJ, 312, 367.

Wielen, R., 1977, A\&A, 60, 263.

Arcadio Poveda and Christine Allen: Instituto de Astronomía, Universidad Nacional Autónoma de México, Apartado Postal 70-264 04510, México, D.F. (poveda@servidor.unam.mx, chris@astroscu.unam.mx). 


\section{DISCUSSION}

Zinnecker - If you say Oepik was right, does that imply that Duquennoy \& Mayor (DM) are wrong, or what's going on?

Poveda - Well I think that observations favor Oepik.

Zinnecker - Oh, but wait a minute, Oepik had much poorer observations than DM, and that is a reason for concern, of course.

Poveda - Well, more modern data keep favoring Oepik.

Zinnecker - What do you mean by more modern data?

Poveda - I mean binaries in the solar vicinity, LDS binaries, WDS binaries. What I say is that from about $50 \mathrm{AU}$ or so, and up to separations of $5000 \mathrm{AU}$, Oepik is a better description of reality than the DM Gaussian distribution, or than Close's power law, as we have shown by means of a Kolmogorov-Smirnov test. For shorter separations I suppose DM is correct, I have nothing to say for smaller separations.

Zinnecker - But this means that the turn-down of the DM distribution in the right half of the log-normal distribution is the part you are actually challenging, you are saying that it has to stay flat.

Poveda - Yes, on the plane where the DM distribution is Gaussian, our distribution (oepik's) is flat. But this is quite consistent with the DM distribution, within their error bars!

$A b t$ - Arcadio, we know that for very young stars like OB stars, there are doubles which have separations of 40000 to $50000 \mathrm{AU}$. Is this simply a matter of age?

Poveda - Well in a certain sense, yes, that means that young stars may form with very wide separations and, if not enough time has gone by relative to their nuclear ages, they will be recognized as bound OB systems. Given enough time they will be broken down by perturbations with massive objects.

Mardling - Can you tell us how Weinberg et al. did their dynamical study of binaries going past massive objects?

Poveda - Essentially, they model the disk of the galaxy adding massive objects with masses of about 1 million or half a million solar masses, moving at random, trying to simulate what is known about the distribution of molecular clouds. Then they model encounters between these massive objects and binaries, and they compute the energy exchange, the gravitational kicks that these binaries experience. I showed two models, one in which the massive perturbing object is a giant cloud with embedded point masses, and one in which the embedded objects are finite clumps of $4 \mathrm{pc}$ radius.

Mardling - It would make the difference whether they treated the massive cloud as an extended object or non-extended object.

Poveda - Yes, indeed. That is why there were two curves for each semimajor axis, one for a point mass and one for an extended object.

Allen - There is a poster by Prieto et al. that shows that the distribution of separations for the Hipparcos double and multiple stars (in a volume-complete sample) also follows Oepik's, out to a maximum separation that is set by instrumental limits of the Hipparcos catalogue. 